\title{
High frequency ultrasonography of the hand versus anti-RA33 evaluation in early rheumatoid arthritis - a pilot study
}

\author{
Andreea Lili Barbulescu', Paulina Lucia Ciurea ${ }^{2}$, Carmen Mitran ${ }^{3}$, Beatrice Andreea Chisalau ${ }^{4}$, \\ Cristina Dorina Parvanescu ${ }^{4}$, Sineta Cristina Firulescu ${ }^{4}$, Maria Balasoiu ${ }^{5}$, \\ Mihail Virgil Boldeanu' ${ }^{6}$, Horatiu Popoviciu ${ }^{7}$, Florentin Ananu Vreju ${ }^{2}$
}

\begin{abstract}
${ }^{1}$ Department of Pharmacology, University of Medicine and Pharmacy, Craiova, ${ }^{2}$ Department of Rheumatology, University of Medicine and Pharmacy, Craiova, ${ }^{3}$ University of Medicine and Pharmacy of Craiova, ${ }^{4}$ Department of Rheumatology, Emergency County Hospital, Craiova, ${ }^{5}$ Department of Microbiology, University of Medicine and Pharmacy, Craiova, ${ }^{6}$ Department of Immunology, University of Medicine and Pharmacy, Craiova, ${ }^{7}$ Department of Rheumatology, University of Medicine and Pharmacy, Targu Mures, Romania
\end{abstract}

\begin{abstract}
Aim: Accurate diagnosis and early treatment in rheumatoid arthritis (RA) can lead to a good outcome and a correct management of the disease. We aimed toinvestigate the prognostic value of anti-RA33 antibodies, by evaluating the relationship with ultrasonographic (US) findings in patients with early RA. Material and methods: We performed a prospective study which included 29 patients, diagnosed with early RA according to the ACR/EULAR 2010 criteria and 21 sex and age-matched control subjects. All patients underwent clinical and biological assessment, followed by US examination in grayscale (GS) and power Doppler (PD) at baseline and after 12 months [from the second to the fifth metacarpophalangeal (MCP) and proximal interphalangeal (PIP) joints and wrists (RCC), in dorsal aspect]. The second and fifth MCP joints were scanned also in lateral aspects. Results: The initial GS evaluation revealed the presence of synovitis in all 29 patients; PD found at least one joint with a PD grade higher than 1 in 23 patients, higher than 2 in 20 patients, and grade 3 in 6 patients; at 12 months, we revealed the presence of GSUS synovitis in 25 patients and PDUS examination found active synovitis in 12 subjects. In those patients, the anti-RA33 titre was significantly lower compared to those without PDUS active synovitis ( $\mathrm{p}=0.031)$, with a moderately negative correlation $(\mathrm{r}=-0.519, \mathrm{p}=0.0039)$. Conclusions: The current study shows that anti-RA33 antibodies might constitute an additional tool for diagnosing early RA patients and can help identify patients with mild disease and a low level of active synovitis.
\end{abstract}

Keywords: early rheumatoid arthritis; anti-RA33 antibodies; ultrasonography; synovitis.

\section{Introduction}

In rheumatoid arthritis (RA) an early diagnosis is essential for an optimal management of the disease [1], with a real impact on the patients' quality of life [2]. An accurate diagnosis encloses to identify antibodies with

Received 26.10.2016 Accepted 06.01.2017

Med Ultrason

2017, Vol. 19, No 2, 166-171

Corresponding author: Florentin Ananu Vreju, Department of Rheumatology

University of Medicine and Pharmacy of Craiova

2 Petru Rares street

200349 Craiova, Romania

Email: florin_vreju@yahoo.com high specificity and sensitivity for both diagnostic and prognostic purpose [2]. Besides anti-cyclic citrullinated peptide (anti-CCP) and rheumatoid factor (RF), already included in 2010 ACR/EULAR diagnostic criteria [3], new serological markers were tested in order to increase the diagnostic specificity. Among these, anti-RA33 antibodies have emerged as a promising tool, due to their absence or rare presence in non-autoimmune rheumatologic conditions or other autoimmune diseases [4]. Besides the utility in the early diagnostic, the anti-RA33 antibodies improve the real-time assessment of disease activity and help to identify different clinical and pathogenic subsets of RA [5]. Thus, by recognizing the patients with unfavourable prognostic factors, the treatment 
can be optimized using a customized therapeutic agenda $[6,7]$. There are many unanswered questions on how new immunological markers change during the evolution and how they impact on disease course, but it has been observed that anti-RA33 antibodies are associated with a relatively mild disease.

New imaging techniques have emerged as sensitive and reproducible tools for diagnosis, monitoring and assessing the prognosis in different area of research, and their good performance has been proved by several studies [8-11]. Ultrasonography (US), an imaging method with several advantages that has made it lately indispensable in daily medical practice, has an unquestionable value in RA, and offered new opportunities by defining and outlining certain pathologic aspects regarding synovial inflammation, the characteristic hallmark of this disease [12-14]. ACR/EULAR 2010 classification criteria acknowledged that US findings can be used for the confirmation of joint involvement, as an essential and accurate tool for assessing the presence of synovitis. Besides its diagnostic utility, US assessment has an important role in monitoring the disease, in which case, US findings may constitute a predictor for the therapeutic response. In patients in clinical remission, US can reveal persistent synovitis, which can be predictive for a relapse and future joint damage. Therefore, in early RA, assessing a real remission is the major objective for every rheumatologist and thus, developing an algorithm for the diagnosis and monitoring, enclosing both US and serological markers, can provide benefit for optimal management.

There is emerging data about US role both in evaluating RA disease activity and the disease progression risk [15] and in the same time, there is evidence that anti-CCP antibodies have a high prognostic value. However, a complex immunologic pattern could help predict treatment response. Thus, we aimed to investigate a possible prognostic value of anti-RA33 antibodies by evaluating the relationship with US findings and to identify a cut-off value for the serum titre in a cohort of patients with early RA.

\section{Material and methods}

We performed a longitudinal, prospective study, which included a cohort of 29 patients, diagnosed with early RA, according to theACR/EULAR 2010 criteria [4] that presented in the Rheumatology Department of Emergency County Hospital Craiova, between March 2014-September 2015, with a duration of the symptoms under 12 months. The control group included 21 sex and age-matched subjects, without acute or chronic inflammatory disease, history of connective tissue, or other autoimmune diseases.
The study was performed in accordance with the Declaration of Helsinki and approved by the Ethics Committee of the University of Medicine and Pharmacy of Craiova. All patients provided their written informed consent, after receiving a standard form for US and biologic samples, which mentioned that the results would be used for research purposes.

\section{Patients assessment}

The patients' evaluation included clinical examination, laboratory tests and US evaluation performed at 0 and 12 months.

Laboratory tests were performed according to the manufacturer's kit indications. In order to determine anti-RA33 antibodies, we used a Human anti-RA33 Assay kit; venous blood samples were centrifuged at 9700 rotations/minute, for 15 minutes, and the serum obtained was stored at $-80{ }^{\circ} \mathrm{C}$ until analyze. Anti-CCP antibodies were determined using fluorescence immunoassay, RF by latex method, $\mathrm{C}$ reactive protein (CRP) by immunoturbidimetry, and erythrocyte sedimentation rate (ESR) byWestergreen.

Functional status was evaluated using the Health Assessment Questionnaire (HAQ) [16] and disease activity by calculating the 28-Disease Activity Score (DAS) [17], with 4 variables.

Synthetic disease-modifying antirheumatic drugs (sDMARDs) were initiated (Methotrexate for 21 patients, Leflunomide for 4 patients, one patient with Sulfasalazine, and 2 with Hydroxychloroquine), along with variable doses of glucocorticoids (between 8 and $32 \mathrm{mg}$ methylprednisolone) in the RA group.

\section{US evaluation}

US was performed from the second to the fifth metacarpophalangeal (MCP) and proximal interphalangeal (PIP) joints and wrists (RC) joints, in dorsal side of both hands. Moreover, the second and fifth MCP joints were scanned also in lateral aspects.

The examiner used a MyLab 25 machine (EsaoteSpA Genoa, Italy) with a $10-18 \mathrm{MHz}$ frequency linear probe, according to EULAR guidelines [18] and noted synovitis both in grayscale (GS) and Power Doppler (PD). PD examinations were carried out using a Doppler frequency of $8.0 \mathrm{MHz}$ and a pulse repetition frequency of $750 \mathrm{~Hz}$ [19]. All examinations were performed by an expert sonographer, blinded for clinical and laboratory data, according to the OMERACT-EULAR consent and OMERACT preliminary definitions [20,21]. The scoring systems for joint inflammation considered both synovial fluid and synovial proliferation, indicative of joint inflammation [15]. MCP, PIP, and RC joint effusion and synovitis were subjectively scored in GS from 0 to $3(0=$ absence; $1=$ mild; $2=$ moderate; $3=$ marked) (fig 1). The 


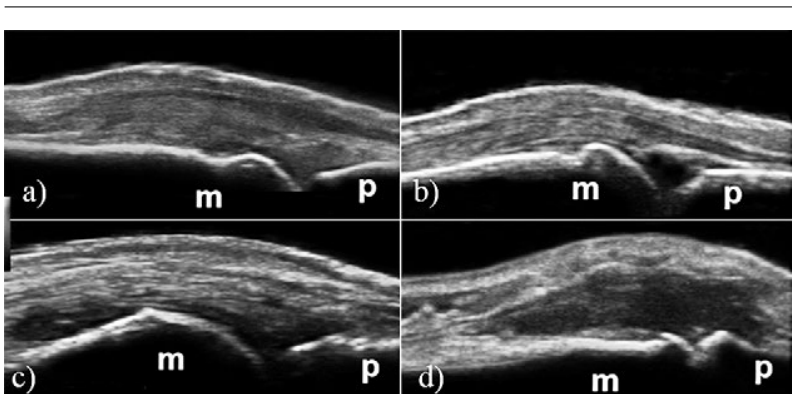

Fig 1. Dorsal longitudinal GS scan showing scoring of the MCP joint synovitis: Grade 0 -absence of synovitis; B. Grade $1=$ mild synovitis; C. Grade 2 = moderate synovitis; D. Grade $3=$ marked synovitis. $\mathrm{m}=$ metacarpal head; $\mathrm{p}=$ proximal phalanx.

intra-articular PD signal was subjectively graded using a semiquantitative scoring system ranging from 0 to 3 ( $0=$ absence, no intra-articular PD signal; $1=$ mild, $P D$ signal due to a single vessel; $2=$ moderate, $P D$ signal due to confluent vessels; $3=$ marked, PD signals in more than half of the intra-articular area).

\section{Statistical Analysis}

Statistical analysis was performed using GraphPad Prism 5.5. Results are presented as mean \pm SD and data were analyzed using t-test and One-way ANOVA for comparing groups, and Pearson/Spearman's coefficient for evaluating correlations. We considered a level of $\mathrm{p}<0.05$ statistically significant.

\section{Results}

The initial clinical and biological findings of the 29 patients included in the study are presented in table I.

RF was positive in 14 patients (48.27\%), with a mean value of $86.65 \pm 105.7 \mathrm{UI} / \mathrm{ml}$, anti-CCP antibodies were positive in 17 patients $(58.62 \%)$, with a mean value of $254.0 \pm 531.3 \mathrm{UI} / \mathrm{ml}$. For anti-RA33 antibodies, we found a mean value of $0.129 \pm 0.130 \mathrm{ng} / \mathrm{ml}$ in the RA group and $0.099 \pm 0.05 \mathrm{ng} / \mathrm{ml}$ in controls $(\mathrm{p}=0.0103)$. We considered the threshold for positivity the mean value calculated for the control group $(0.099 \pm 0.05 \mathrm{ng} / \mathrm{ml})$, after cutting out the minimum and maximum values. Thus, 14 patients $(48.27 \%)$ were considered to be positive for anti-RA33 antibodies; hence, the diagnostic sensitivity was $40 \%$, the specificity $90 \%$, with a positive predictive value of 0.875 , and a negative predictive value of 0.475 . The frequency of anti-RA33 antibodies between patients with RFpositive, was $21.42 \%$ ( 4 cases), while anti-CCP antibodies were present in 9 cases $(64.42 \%)$. We found a moderately positive correlation between the RF and anti-CCP antibodies titres $(r=0.47, p=0.0097)$, while for anti-RA33, we found no statistical significant relationship with the presence of RF or anti-CCP antibodies.
Table I. Baseline characteristics of the patients

\begin{tabular}{ll}
\hline Characteristics & \\
\hline Age (years) & $41.72+9.38$ \\
Sex (women) & $28(96.55)$ \\
Disease duration (months) & $6.72+2.55$ \\
ESR (mm/h) & $48.76+37.27$ \\
CRP $(\mathrm{mg} / \mathrm{l})$ & $12.37+1.85$ \\
anti-RA33 (ng/ml) & $0.139+0.138$ \\
RF $(\mathrm{UI} / \mathrm{ml})$ & $86.65+105.7$ \\
RF positive & $14(48.27)$ \\
anti-CCP $(\mathrm{UI} / \mathrm{ml})$ & $254.0+531.3$ \\
anti-CCP positive & $17(58.62)$ \\
TJC & $8.24+1.88$ \\
SJC & $3.75+1.52$ \\
VASp & $66.20+1.65$ \\
VASm & $59.31+7.22$ \\
DAS28 (4v) & $4.61+0.76$ \\
HAQ & $1.31+0.53$ \\
\hline
\end{tabular}

The results are expressed asa percentage $(\mathrm{n} \%)$ or mean \pm standard deviation. ESR-erytrocyte sedimentaton rate; $\mathrm{CRP}-\mathrm{C}$ reactive protein; RF-rheumatoid factor; anti-CCP anti cyclic citrullinated peptide; TJC-tender joint count; SJC-swollen joint count; VASp-visual analogue scale patient; VASm- physician's visual analogue scale; $\operatorname{DAS}_{(4 \mathrm{v})}$-disease activity index with 28 joints; HAQ-health assessment questionnaire.

At the moment of diagnosis, we evaluated the disease activity, using the DAS28 $8_{(4 \mathrm{v})}(\mathrm{CRP})$ scoring and we reckoned a mean value of $4.61 \pm 0.76(\min 2.99$; $\max$ $6.29)$ in the RA patients group; most of the patients (24; $82.7 \%$ ) had a moderate disease activity, while 4 of them $(13.79 \%)$ registered a value corresponding to a high disease activity and only one was in low disease activity.

The initial GSUS evaluation revealed the presence of at least one joint with a synovitis score higher than 1 in all 29 patients; using the PDUS score, we found 23 patients with at least one joint with a score higher than 1,20 patients with score higher than 2, and 6 patients with grade 3 .

After 12 months, using DAS28 ${ }_{(4 \mathrm{v})}(\mathrm{CRP}), 17$ patients $(58.62 \%)$ registered a low disease activity, $8(27.58 \%)$ were in remission and $3(10.34 \%)$ had a moderate disease activity. Anti-RA33 titre was significantly different between the three groups $(0.143 \pm 0.1786 \mathrm{ng} / \mathrm{ml} ; 0.153 \pm 0.073 \mathrm{ng} /$ $\mathrm{ml}$; respectively $0.087 \pm 0.094 \mathrm{ng} / \mathrm{ml}, \mathrm{p}=0.044)$. Analyzing the response, we established a medium delta DAS of 1.79+0.86; therefore, 7 patients $(24.13 \%)$ registered a moderate EULAR response, 2 (6.89\%) had no response and $20(68.96 \%)$ a good response. We found a moderate positive correlation $(\mathrm{r}=0.4566, \mathrm{p}=0.0128)$ between the initial titre of anti-RA33 antibodies and delta DAS (fig 2).

US examination at 12 months revealed the presence of GS synovitis in 25 patients with a mean number of interested joints in the whole study group of $1.58 \pm 1.27$ 
(limits 0-8); PDUS examination found active synovitis for 12 subjects, with a mean PDUS score of $2.24 \pm 3.02$; all scores higher than 2 were found in patients with an anti-RA33 titre lower than $0.99 \mathrm{ng} / \mathrm{ml}$.

Analysing the anti-RA33 titre, depending on the GSUS findings, we found a slightly higher value $(0.144 \pm 0.151 \mathrm{ng} / \mathrm{ml})$, but without statistical significance $(\mathrm{p}=0.377)$ in patients with synovitis (GSUS+) in comparison to patients without synovitis (GSUS -) $(0.105 \pm 0.026 \mathrm{ng} / \mathrm{ml})$. At the same time, anti-RA33 titre was $0.107 \pm 0.056 \mathrm{ng} / \mathrm{ml}$ for patients with active synovitis, significantly lower compared to the second group, without PDUS synovitis $(0.159 \pm 0.177 \mathrm{ng} / \mathrm{ml}, \mathrm{p}=0.031)$ (table II, fig 3 ). Also between the 14 patients with an anti-RA33 titre higher than $0.099 \mathrm{ng} / \mathrm{ml}$, only 3 had active synovitis.

When evaluating the disease activity in relation to the anti-RA33 antibodies titre, we found the same tendency for negative correlation between the antibodies titre,

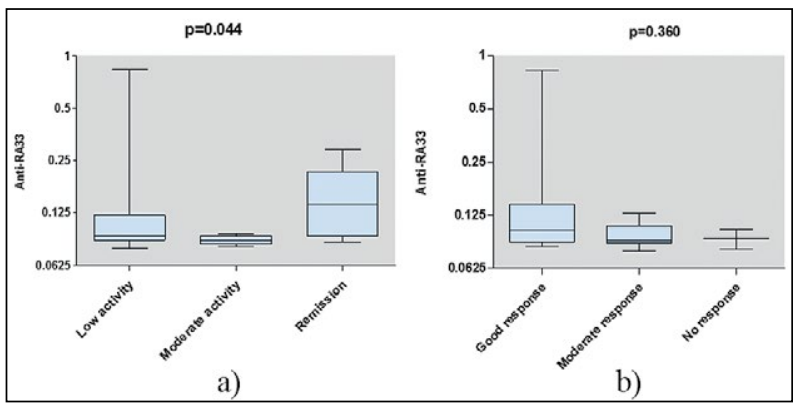

Fig 2. Anti-RA33 titre (ng/ml) depending on a) disease activity and b) EULAR response

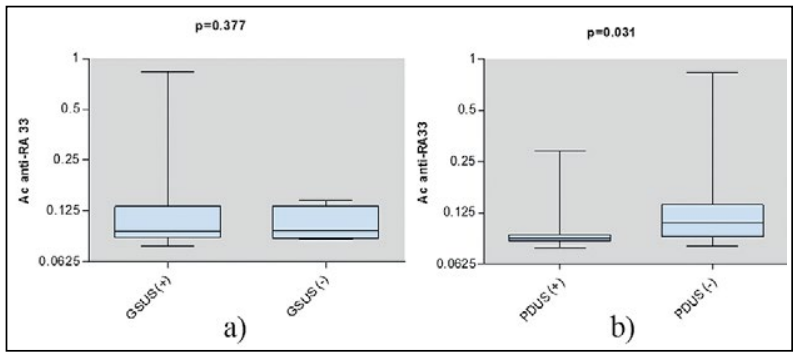

Fig 3. Anti-RA33 antibodies $(\mathrm{ng} / \mathrm{ml})$ depending on the presence/absence of GSUS/PDUS synovitis
DAS28, and PDUS scores, with a higher difference for the last one.

Anti-CCP antibodies had different values in relationship to the presence/absence of GSUS synovitis $(703.3 \pm 1226 \mathrm{UI} / \mathrm{ml} v s .139 .4 \pm 212.7 \mathrm{UI} / \mathrm{ml})$ but with no statistical significance $(\mathrm{p}=0.490)$. PDUS active synovitis revealed a titre of anti-CCP antibodies of $961 \pm 1179 \mathrm{UI} /$ $\mathrm{ml} v s .125 .6 \pm 191.7 \mathrm{UI} / \mathrm{ml}$ for patients without PDUS active synovitis $(\mathrm{p}=0.0051)$.

We found a moderately negative correlation $(\mathrm{r}=-$ $0.519, \mathrm{p}=0.0039$ ), between the baseline titre of antiRA33 antibodies and the presence of US active synovitis after 12 months of treatment. Also, for anti-CCP antibodies and RF we established a moderately positive correlation coefficient ( $\mathrm{r}=0.412$ and $\mathrm{r}=0.472$, respectively).

Patients that needed therapy with two conventional sDMARDs $(6 ; 20.68 \%)$ at 12 months, had a baseline titre of anti-RA33 antibodies significantly lower compared to the subjects that needed only one sDMARD $(23 ; 79.31 \%)$ $(088 \pm 0.0111 \mathrm{ng} / \mathrm{ml}$ vs $0.151 \pm 0.156 \mathrm{ng} / \mathrm{ml}, \mathrm{p}=0.013)$.

\section{Discussions}

Setting an antibody profile, highly specific and sensitive, with a diagnostic and prognostic value, could be essential for assessing remission[1,22-26]. Besides anti-CCP antibodies, present very early in the course of the disease, anti-RA33 antibodies can constitute an additional marker, not only for improving diagnosis, but also for assessing the prognosis and therapeutic response $[5,27,28]$.

The reported percentage of positive cases for antiRA33 antibodies varies between studies [3,5,29-31]. In our cohort almost half of the patients were positive. Consistent to our data, a recent meta-analysis, which included studies performed between 2000-2015 concerning the diagnostic value of anti-RA33 antibodies in RA, showed similar results [5]. It is also noteworthy that for positive RF patients, our results showed a low percentage of anti-RA33 positivity, while more than half of the antiCCP positive subjects were anti-RA33 positive. Similar data were reported by Nell et al [6], in a cohort of 102 early RA subjects, and also by Al-Mughales et al [29].

Table II. Anti-RA33 and anti-CCP titre depending on the presence/absence of synovitis in grey scale and power Doppler ultrasonography.

\begin{tabular}{|c|c|c|c|c|}
\hline & GSUS + & GSUS - & PDUS + & PDUS - \\
\hline Anti-RA33 (ng/ml) & $0.105+0.151$ & $0.144+0.026$ & $0.107+0.056$ & $0.159+0.177$ \\
\hline $\mathrm{p}$ & 0.377 & & 0.031 & \\
\hline Ac anti-CCP $(\mathrm{UI} / \mathrm{ml})$ & $703.3+1226$ & $139.4+212.7$ & $961+117$ & $125.6+191.7$ \\
\hline $\mathrm{p}$ & 0.490 & & 0.005 & \\
\hline
\end{tabular}

GSUS-grey scale ultrasonography, PDUS- power Doppler ultrasonography, + present, - absent. 
This finding underlines the necessity and benefit of determining anti-RA33 antibodies in RF seronegative RA patients.

Analysing disease activity over the 12 months, our results revealed a highly divergent, statistically significant, anti-RA33 titre between patients with low disease activity, moderate activity, and remission.Moreover, when assessing the improvement of DAS28 ${ }_{(4 \mathrm{v})}$ score and anti-RA33 titre, we found a moderate positive correlation, statistically significant between the two variables. The data are consistent with other studies which concluded that the presence of anti-RA33 characterises patients with mild disease [6].

Persistent synovitis, especially in patients with early and very early RA, was proven to be present in patients being considered in clinical remission and was associated with a more unfavourable prognosis [15,22,26,32]. Analysing anti-RA33 titre, depending on US findings, we established statistically significant differences, between patients with/without active synovitis; thus, we found active synovitis only in 3 of the 15 patients with an anti-RA33 titre over the threshold value. A moderate, negative correlation between the two variables was established.

In agreement with these findings that underline the protective role of higher anti-RA33 antibodies titre, we noticed that for patients who received two conventional sDMARDs, the titre of anti-RA33 antibodies was significantly lower, compared to the subjects receiving one sDMARD.

Regarding anti-CCP antibodies, they have been proven to be directly related to a more erosive and progressive disease, with an important impact on future articular damage and, consequently on the evolution and prognosis. Analysing US findings, we found a moderately positive correlation, but not statistically significant, between the initial titre of anti-CCP antibodies and the presence of active synovitis, after 12 months of treatment. A similar observation was noted by Harman et al [1].

Both GSUS and PDUS are important in order to discriminate between the clinical remission with US subclinical inflammation and the real one, with no sign of US active synovitis, and can constitute the base of a prediction model that integrates several outcome measures. As it was shown by our results, patients with high titres of anti-RA33 antibodies had a milder evolution, with a low degree of US inflammation. These results led to the idea that remission can be predicted by a combination of clinical and immunological items and confirmed by US.

The main limitations of the study are as follows: firstly, the low number of patients, with the prevalence of younger female patients, a fact that might restrict some- how the practical applicability of the results in a general RA population, without further extension of the group; in addition, the number of patients in which we established the cut-off value is low. Secondly, we acknowledge the fact that the US evaluation was performed by a single examiner, even if expert, using a machine with some technical limitations, especially on PDUS sensitivity and also that we had not performed other imaging techniques, such as MRI, to confirm our findings. Finally, the study design permits the different types of treatment in patients, which could influence the disease outcome.

\section{Conclusions}

The current preliminary study shows that anti-RA33 antibodies might constitute an additional tool for diagnosing early RA patients, with a particular contribution in RF seronegative cases. Also, in our cohort, the presence of anti-RA33 antibodies identified patients with a mild disease and with a very low level of active synovitis assessed by PDUS. Despite the relatively low number of subjects included in the study, the results are in agreement with other recently published papers and calls for its extension, with multicentre contribution and larger cohort of patients with early and very early RA. Determining additional antibodies, specific for a disease, with an important impact on the evolution of the patients, and integrating the results with ultrasound findings, not only improves the diagnosis, but can also help quantify the future articular damage and outcome of the patients.

\section{Conflict of interest: none}

\section{References}

1. Harman H, Tekeoglu I, Kaban N, Harman S. Factors influencing ultrasonographic remission in patients with rheumatoid arthritis. Rheumatol Int 2015;35:485-491.

2. Traistaru MR, Kamal D, Trasca DM, Foarfa MC, Gruia CL, Rogoveanu OC. Rheumatoid nodules and quality of life in rheumatoid arthritis females - complex assessment. Rom J Morphol Embryol 2016;57:215-225.

3. Conrad K, Roggenbuck D, Reinhold D, Dörner T. Profiling of rheumatoid arthritis associated autoantibodies. Autoimmun Rev 2010;9:431-435.

4. Aletaha D, Neogi T, Silman AJ, et al. 2010 Rheumatoid arthritis classification criteria: an American College of Rheumatology/European League Against Rheumatism collaborative initiative. Arthritis Rheum 2010;62:2569-2581.

5. Yang X, Wang M, Zhang X, et al. Diagnostic accuracy of anti-RA33 antibody for rheumatoid arthritis: systematic review and meta-analysis. Clin Exp Rheumatol 2016;34:539547. 
6. Nell VP, Machold KP, Stamn TA, et al. Autoantibody profiling as early diagnostic and prognostic tool for rheumatoid arthritis. Ann Rheum Dis 2005;64:1731-1736.

7. Rogoveanu OC, Mogoşanu GD, Bejenaru C, et al. Effects of Calcium Fructoborate on Levels of C-Reactive Protein, Total Cholesterol, Low-Density Lipoprotein, Triglycerides, IL1 $\beta$, IL-6, and MCP-1: a Double-blind, Placebo-controlled Clinical Study. Biol Trace Elem Res 2015;163:124-131.

8. Dumitrescu CI, Gheonea IA, Săndulescu L, Surlin V, Săftoiu A, Dumitrescu D. Contrast enhanced ultrasound and magnetic resonance imaging in hepatocellular carcinoma diagnosis. Med Ultrason 2013;15:261-267.

9. Gheonea IA, Donoiu L, Camen D, Popescu FC, Bondari S. Sonoelastography of breast lesions: a prospective study of 215 cases with histopathological correlation. Rom J Morphol Embryol 2011;52:1209-1214.

10. Gheonea IA, Streba CT, Cristea CG, et al. MRI and pathology aspects of hypervascular nodules in cirrhotic liver: from dysplasia to hepatocarcinoma. Rom J Morphol Embryol 2015;56:925-935.

11. Ungureanu BS, Pirici D, Margaritescu C, et al. Endoscopic ultrasound guided injection of iron oxide magnetic nanoparticles for liver and pancreas: a feasibility study in pigs. Med Ultrason 2016;18:157-162.

12. Senolt L, Grassi W, Szodoray P. Laboratory biomarkers or imaging in the diagnostics of rheumatoid arthritis? BMC Med 2014;12:49.

13. Vreju F, Ciurea M, Roşu A, Muşetescu A, Grecu D, Ciurea P. Power Doppler sonography, a non-invasive method of assessment of the synovial inflammation in patients with early rheumatoid arthritis. Rom J Morphol Embryol 2011;52:637-643.

14. Vreju FA, Chisalau BA, Parvanescu CD, Ciurea P. High Frequency Ultrasonography of the Hand in Rheumatoid Arthritis, Psoriatic Arthritis, Gout and Osteoarthritis Patients. Curr Health Sci J 2016;42:35-39.

15. Vreju FA, Filippucci E, Gutierrez M, et al. Subclinical ultrasound synovitis in a particular joint is associated with ultrasound evidence of bone erosions in that same joint in rheumatoid patients in clinical remission. Clin Exp Rheumatol 2016;34:673-678.

16. Bruce B, Fries JF. The Health Assessment Questionnaire (HAQ). Clin Exp Rheumatol 2005;23(5 Suppl 39):S14-S18.

17. Wells G, Becker JC, Teng J, et al. Validation of the 28-joint Disease Activity Score (DAS28) and European League Against Rheumatism response criteria based on C-reactive protein against disease progression in patients with rheumatoid arthritis, and comparison with the DAS28 based on erythrocyte sedimentation rate. Ann Rheum Dis 2009;68:954-960.

18. Backhaus M, Burmester GR, Gerber T, et al; Working Group for Musculoskeletal Ultrasound in the EULAR Standing Committee on International Clinical Studies including Therapeutic Trials. Guidelines for musculoskeletal ultrasound in rheumatology. Ann Rheum Dis 2001;60:641-649.
19. Torp-Pedersen ST, Terslev L. Settings and artefacts relevant in colour/power Doppler ultrasound in rheumatology. Ann Rheum Dis 2008;67:143-149.

20. Naredo E, Wakefield RJ, Iagnocco A, et al. The OMERACT ultrasound task force-status and perspectives. J Rheumatol 2011;38:2063-2067.

21. Wakefield RJ, Balint PV, Szkudlarek M, et al; OMERACT 7 Special Interest Group. Musculoskeletal ultrasound including definitions for ultrasonographic pathology. J Rheumatol 2005;32:2485-2487.

22. Naredo E, Collado P, Cruz A, et al. Longitudinal power Doppler ultrasonographic assessment of joint inflammatory activity in early rheumatoid arthritis: predictive value in disease activity and radiologic progresssion. Arthritis Rheum 2007;57:116-124.

23. Kawashiri SY, Kawakami A, Iwamoto N, et al. The power Doppler ultrasonography score form 24 synovial sites or 6 simplified synovial sites, including the metacarpophalangeal joints, reflects the clinical disease activity and level of serum biomarkers in patients with rheumatoid arthritis. Rheumatology (Oxford) 2011;50:962-965.

24. Chakr RM, Mendonca JA, Brenol CV, Xavier RM, Brenol JC. Assessing rheumatoid arthritis disease activity with ultrasound. Clin Rheumatol 2013;32:1249-1254.

25. Damjanov N, Radunovic G, Prodanovic S, et al. Construct validity and reliability of ultrasound disease activity score in assessing joint inflammation in RA: comparison with DAS-28. Rheumatology (Oxford) 2012;51:120-128.

26. Scire CA, Montecucco C, Codullo V, Epis O, Todoerti M, Caporali R. Ultrasonographic evaluation of joint involvement in early rheumatoid arthritis in clinical remission: power Doppler signal predicts short-term relapse. Rheumatology (Oxford) 2009;48:1092-1097.

27. Trouw L, Mahler M. Closing the serological gap: promising novel biomarkers for the early diagnosis of rheumatoid arthritis. Autoimmun Rev 2012;12:318-322.

28. Maslyanskiy A, Lazareva N, Olinek P, et al. Anti-hnRNP B1 (RA33) autoantibodies are associated with the clinical phenotype in Russian patients with rheumatoid arthritis and systemic sclerosis. J Immunol Res 2014;2014: 516593.

29. Al-Mughales JA. Immunodiagnostic significance of antiRA33 autoantibodies in Saudi patients with rheumatoid arthritis. J Immunol Res 2015;2015:604305.

30. Al-Ani MM. Comparison of anti-filaggrin, anti-RA33 and anti-cyclic citrullinated peptide antibodies in the diagnosis of rheumatoid arthritis in Iraqi patients. Iraqi J Comm Med 2013;3:258-261.

31. Lashkari M, Noori A, Hajiimanouchehri F, Oveisi S, Kazemifar AM. Determination of specificity and sensitivity of anti-RA33 in diagnosis of early rheumatoid arthrtis. Glob J Health Sci 2014;6:292-297.

32. Peluso G, Michelutti A, Bosello S, Gremese E, Tolusso B, Ferraccioli G. Clinical and ultrasonographic remission determines different chances of relapse in early and long standing rheumatoid arthritis. Ann Rheum Dis 2011;70:172-175. 